\section{Sleep terror disorder: a case report} Terror noturno: um relato de caso
Carlos Simon Guzman, Yuan Pang Wang Institute of Psychiatry, Universidade de São Paulo (USP), São Paulo (SP), Brazil
Dear Editor,

We report a case of sleep terror in a 4-year-old boy, patient A. The parents observed that for the past month, after the patient going bed to sleep, A. have being wake up in the middle of the night. This behavior occurs once or twice a week. On these occasions, the child is found standing somewhere in the house, crying and seemingly disoriented with rapid breathing and profuse sweating. When the parents attempt to comfort him or return him to his room, he becomes quite upset, striking out at them and screaming loudly. He continues to scream and fight for several minutes, followed by spontaneous cessation. Once the child is calmed, the parents can put him back in his bed, and he sleeps through the rest of the night without incident. In the morning, he wakes up in usual happy mood and does not remember what occurred the previous evening. The parents are worried that the might be having seizures or developing a severe behavioral problem. Lab blood work-up is solicited, including an electroencephalogram (EEG). All returned normal results.

Arousal parasomnias (night terrors, sleepwalking, and confusional arousals) have seldom been investigated in the adult general population. Clinical studies of parasomnias, however, show that these disorders may be indicators of underlying mental disorders and may have serious consequences. Prevalence rates are based on self-reported data and, consequently, are likely underestimated. ${ }^{1,2}$

Night terror and somnambulism tend to occur during periods of arousal from delta sleep. As a result, these disorders are more common in children who have more delta sleep and they often occur during the first half of the night when there is more delta sleep occurring. Patient who sleepwalks often shows amnesia to the event on awakening as well. ${ }^{2}$

Generally, pharmacologic or extensive behavioral treatment should not be considered prior to age 7 . The treatment of sleep terror disorder usually consists of reassuring the parent that, with time, the child will outgrow these harmless events. Nocturnal enuresis might be involved with night terror event. ${ }^{1-3}$ Our patient A also presented about one year ago a history of enuresis that was successfully treated by imipramine. However, the recommended treatment for sleep terror disorder is to assure the parents that their child will probably grow out of this developmental, but in this case we chose a selective serotonin reuptake inhibitor antidepressant - fluoxetine $20 \mathrm{mg}$ per day, for 2 months, achieving good response after 6 weeks treatment. ${ }^{4}$

This patient's presentation is typical for sleep terror, a disorder that found in $3 \%$ of all children and less than $1 \%$ of adults. Typically, sleep terror manifests itself as emotional an behavioral disturbances at night. This disorder is usually time-limited. ${ }^{4,5}$ These findings suggest that specific factors, perhaps reflecting an interaction of lifestyle and hereditary contributions, may be responsible for the observed variability in this condition. ${ }^{4}$ More research to clarify the underlying physiopathology of this disorder is important to define the differential diagnosis and test the efficacy of different treatment.
Financial support: None

Conflict of interests: None

References

1. Ohayon MM, Guilleminault C, Priest RG. Night terrors, sleepwalking, and confusional arousals in the general population: their frequency and relationship to other sleep and mental disorders. J Clin Psychiatry. 1999;60(4):268-76.

2. Cartwright R. Sleepwalking violence: a sleep disorder, a legal dilemma, and a psychological challenge. Am J Psychiatry. 2004;161(7):1149-58.

3. Bader G, Nevéus T, Kruse S, Sillén U. Sleep of primary enuretic children and controls. Sleep. 2002;25(5):579-83.

4. Gau SF, Soong WT. Psychiatric comorbidity of adolescents with sleep terrors or sleepwalking: a case-control study. Aust N Z J Psychiatry. 1999;33(5):734-9.

5. Kales A, Soldatos CR, Bixler EO, Ladda RL, Charney DS, Weber G, Schweitzer PK. Hereditary factors in sleepwalking and night terrors. Br J Psychiatry. 1980;137:111-8.

\section{Cold weather and risk of sudden cardiac death in schizophrenia: finding a new Achilles' heel?} Baixas temperaturas e risco de morte súbita cardíaca na esquizofrenia: desvendando um novo calcanhar de Aquilles?

\section{Dear Editor,}

People with schizophrenia have a two to three-fold increased risk to die prematurely than those without schizophrenia and this excess of mortality is accounted for by a combination of increased risk factors such as patients' life style, suicide (in particular in young male patients soon after diagnosis), premature development of cardiovascular disease, high prevalence of metabolic syndrome, carbohydrate and lipid metabolic disorders and equally important but not so often mentioned is sudden unexpected death. ${ }^{1}$ The exact pathophysiological ca use of sudden unexpected death in schizophrenia (SUDS) is unknown, but it is probable that cardiac arrhythmia plays a potential role. ${ }^{2}$ Because cardiac abnormalities are an important cause in sudden death we discuss whether it is possible that winter temperatures may facilitate cardiac abnormalities and hence SUDS. Exposure to winter temperatures is considered to be one of the main factors influencing morbidity and mortality from cardiovascular 